\title{
Porfirinas en Cálculos Renales (Revisión)
}

\author{
M는. Traba Villameytide \\ Bioquímica Clínica. Sección Fisiopatología Ósea. Fundación Jiménez Díaz. Madrid. \\ Actas Urol Esp 2005; 29 (2): 163-169
}

\section{RESUMEN}

PORFIRINAS EN CÁLCULOS RENALES (REVISIÓN)

Hemos realizado una revisión del contenido de porfirinas en una clase peculiar de cálculos renales. Estos son negros como el carbón y presentan un espectro infrarrojo (EIR) similar al conocido como "materia orgánica", el cual no indica la clase de sustancia a la que corresponde. Otros tipos de cálculos renales, principalmente de pequeño tamaño y eliminados espontáneamente, pueden tener un compuesto oscuro o negro de forma difusa o aislada y presentan un espectro de IR similar al de "materia orgánica". Después de observar que la hemoglobina presentaba un espectro de IR de "materia orgánica”, estudiamos, por cromatografía en capa fina, un método sensible para la determinación de porfirinas, la presencia de porfirinas en diversos tipos de cálculos renales negros y oscuros, ya que las porfirinas son los precursores del grupo hemo. Este trabajo ha puesto de manifiesto dos tipos de porfirinas: la coproporfirina, en pacientes que padecen hepatopatías, y la uroporfirina y heptacarboxilporfirina en pacientes con algún tipo de porfiria o bien con fracaso renal crónico.

Palabras clave: Cálculos renales negros. Coproporfirina. Uroporfirina.

\section{ABSTRACT}

PORPHYRINS IN RENAL CALCULI (REVIEW)

We performed a review of the porphyrines content in a type of black, charcoal-like, renal calculi exhibiting infrarred spectra (IRS) similar to those characteristic of "organic material" which has not yet been fully elucidate. Several other types of renal calculi, mainly those of small size, spontaneous passage renal stone, may also have diffuse or isolated dark charcoal components showing "organic material" IRS. After observing that haemoglobin has an "organic material" IRS, we studied, by a sensitive thin layer chromatography method, the presence of porphyrines in several types of dark or charcoal renal stones, since porphyrines are the physiological precursors of haemo group biosynthesis.

We found two types of porphyrine content: coproporphyrin, in patients suffering from hepatopathy, and uroporphyrin and heptacarboxil-porphyrin in patients with some types of porphyria or with chronic renal failure.

Key words: Porphyrin renal black calculi. Coproporphyrin. Uroporphyrin.

$\mathrm{E}$ xiste un reducido número de cálculos renales negros, generalmente de pequeñas dimensiones, que a la lupa binocular muestran la apariencia del carbón, Fig. 1-I (a, b), y que por espectrometría de infrarrojos (IR) muestran el espectro denominado "materia orgánica", Fig. 2A. Existen otro grupo de pequeños cálculos de expulsión espontánea de Weddellita y Whewellita que morfológicamente y a la lupa binocular muestran un componente negro en forma difusa o aislada en áreas del cálculo, Fig. 1-II (a, b, c, d). El análisis por IR de la materia negra mostró el espectro de "materia orgánica”, Fig. 2 (A, B). Existe otro grupo de cálculos farmacológicos compuestos por triamterene que presentaban un color negruzco, en lugar del característico color dorado, (Fig. 1-III). También nos llamó la atención un grupo de cálculos papilares de Whewellita de color oscuro (Fig. 1-IV). 

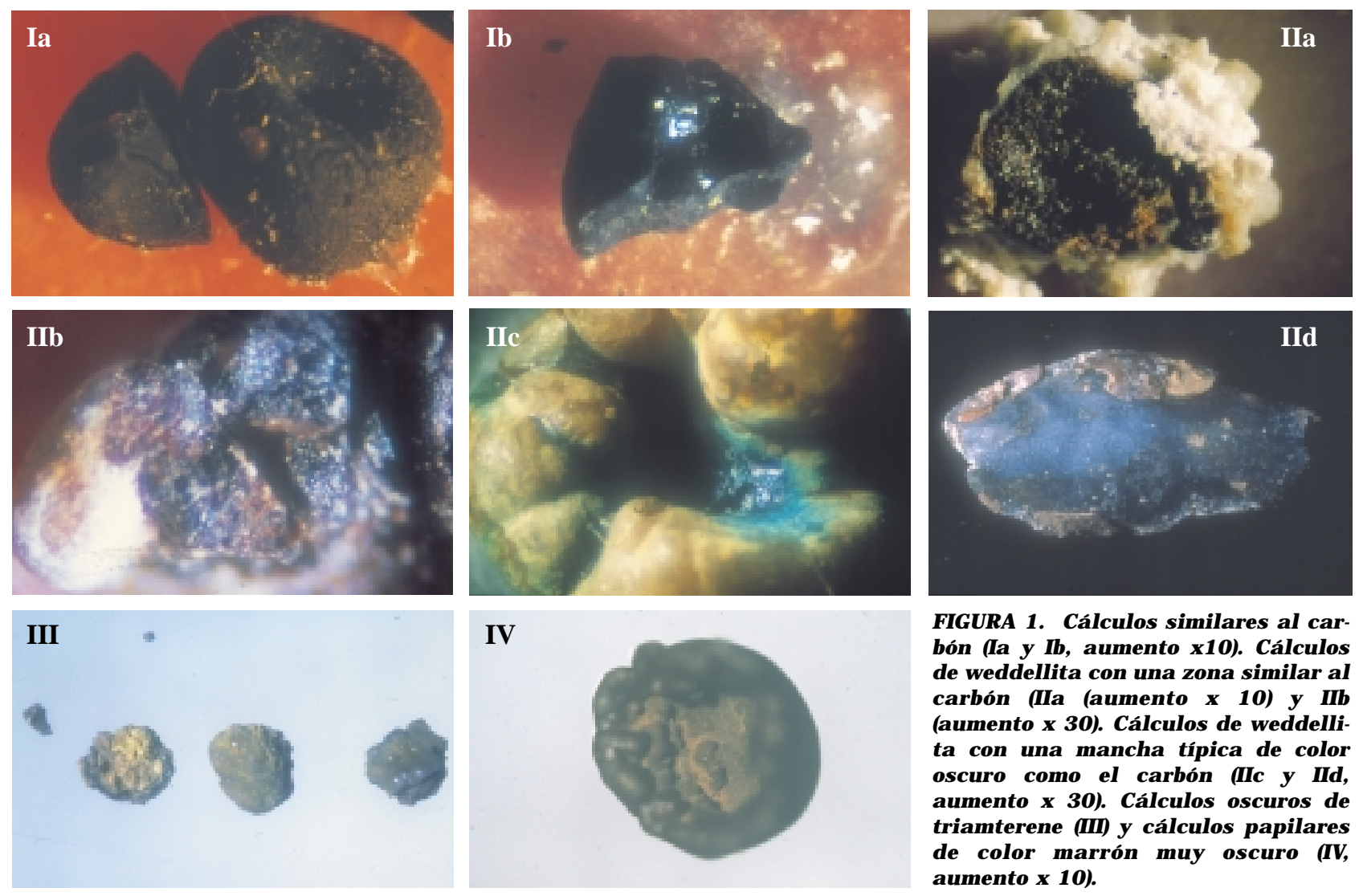

FIGURA 1. Cálculos similares al carbón (Ia y Ib, aumento x10). Cálculos de weddellita con una zona similar al carbón (IIa (aumento $x$ 10) y IIb (aumento $x$ 30). Cálculos de weddellita con una mancha tipica de color oscuro como el carbón (IIc y IId, aumento $x$ 30). Cálculos oscuros de triamterene (III) y cálculos papilares de color marrón muy oscuro (IV, aumento $x$ 10).

Con el fin de definir la composición de los cálculos, éstos fueron diluidos en agua destilada y se aplicaron las tiras de Labstix (Lab Ames, Madrid), dando positiva la reacción de los grupos tetrapirrólicos ${ }^{1}$. A partir de estos resultados nos planteamos realizar un espectro de infrarrojos de hemoglobina (Difco, Difco Laboratories, Detroit, Michigan, USA), de albúmina humana, fracción V de Miles Martín (Lab Ames, Milán, Italia). Ambos mostraron un EIR similar a "materia orgánica", Fig. 2 (D y E respectivamente). La Fig. 2F muestra el espectro del triamterene. También se hicieron espectros de fibrina y globina que muestran espectros mas planos ${ }^{1}$. Estos hechos nos llevaron a la determinación de porfirinas en otro tipo de cálculos negros o bien muy oscuros.

\section{MATERIALES Y MÉTODOS}

De 4.761 cálculos renales analizados por espectroscopía infrarroja, 40 de ellos presentaban el tipo peculiar negro, semejante al carbón (0.8\%) mostrando un EIR de "materia orgánica" 1 ${ }^{4}$ (Fig 2B). En primer lugar, se hizo el análisis de porfirinas ${ }^{5}$ a los grupos de cálculos, Fig 1 (I, II, III)) anteriormente citados, y a un grupo tomado como control formado por cálculos de carbonato apatita, estuvita, whewellita, weddellita y cistina $^{4,6,7}$. Con posterioridad se realizó el contenido de porfirinas de un grupo de cálculos papilares (Fig. 1-IV).

El análisis por espectrometría de infrarrojos se realizó por el método de comprimidos de bromuro potásico ${ }^{3}$ y la determinación de porfirinas por cromatografía de capa fina ${ }^{5}$. Para ello los cálculos fueron homogeneizados (en mortero de vidrio) y sus porfirinas esterificadas a temperatura ambiente y en oscuridad mediante la mezcla metanol/ácido sulfúrico (9:1 v/v). A las 24 h los ésteres de porfirinas fueron extraídos con cloroformo, que una vez lavado y neutralizado fue evaporado. Las muestras se suspendieron en cantidades conocidas de cloroformo y aplicadas a placas cromatográficas de sílicagel G-60 de $0.25 \mathrm{~mm}$ de espesor (Merck) sin indicador de fluorescencia. El desarrollo cromatográfico se efectuó mediante el solvente constituído por acetato de 


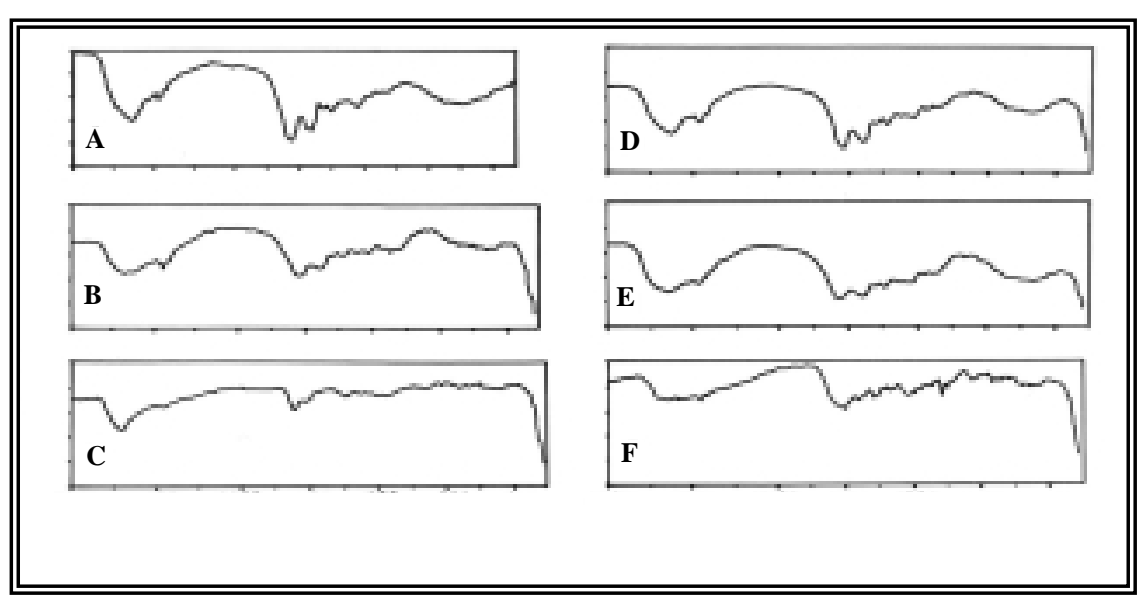

FIGURA 2. Espectros de infrarrojos de: $A$, materia orgánica; $B$, cálculo negro; $C$, mezcla de porfirinas (copro, proto y uroporfirinas); $D$, hemoglobina; E, albúmina y F, cálculos de traimterene.

etilo/propionato de etilo/diclorometano/tetracloruro de carbono (1:1:2:2, v/v). Los cromatogramas se cometiieron a "scanning" fluorométrico en un lector de placas Schimadzu Mod CS-920. La aplicación simultánea de ésteres de porfirinas patrones (Porphyrin Products, Utah), en cantidades conocidas, permitió cuantificar e identificar los metil-ésteres de los diversos carboxil-porfirinas presentes en las muestras de los cálculos a analizar. Por otra parte, los cálculos del grupo I fueron estudiados por el método de cromatografía en capa fina con microscopía de polarización y con microscopía electrónica de barrido.

Dado que las porfirinas tienen la capacidad de unirse a iones para originar metaloporfirinas y debido a la alta reactividad de los grupos carboxílicos de las cadenas laterales pueden unirse a proteínas y lípidos ${ }^{8}$. Por todo ello, a 4 cálculos negros y 4 cálculos controles se les determinó el contenido de iones por espectrometría de absorción atómica (Perkin Elmer), fósforo por el método de Fiske y Subbarow ${ }^{9}$ proteínas totales por el método de Bradford et $\mathrm{al}^{10}$.colesterol por el método de Richmond ${ }^{11} \mathrm{y}$ triglicéridos por el método de Bucolo et al ${ }^{12}$.

El método estadístico aplicado en el estudio de las porfirinas de los grupos I, II, III con respecto al grupo control fue el test no paramétrico de Mann-Whitney. La hipótesis nula fue eliminada para valores de $\mathrm{p}<0.05$. En los demás estudios realizados se aplicó el test de la t de Student. El valor de $\mathrm{p}<0.05$ se consideró significativo.

\section{RESULTADOS}

El grupo control presentó cantidades mínimas de porfirinas $(0.4 \pm 0.87 \mathrm{ng} / \mathrm{mg}$, media \pm $\mathrm{SD}, \mathrm{n}=21$ ) mientras que en los tres grupos de cálculos I, II y III se observó un aumento significativo de porfirinas con respecto al grupo control (grupo I: $74.7 \pm 146.3 \mathrm{ng} / \mathrm{mg}, \mathrm{n}=8$, $\mathrm{p}<0.00003$ vs grupo control; grupo II: $25.1 \pm 33.4 \mathrm{ng} / \mathrm{mg}$, $\mathrm{n}=4, \mathrm{p}<0.027$; grupo III: $8.8 \pm$ $8.55 \mathrm{ng} / \mathrm{mg}, \mathrm{n}=7, \mathrm{p}<0.0013)$ (Tabla I) ${ }^{6}$.

La Tabla II muestra el contenido de porfirinas de un grupo de cálculos papilares. La figura 3 muestra el contenido total de proteínas $(\mu \mathrm{g} / \mathrm{mg})$ en los grupos de cálculos I,II,III y IV y control.

Se han observado dos patrones de acumulación de porfirinas. En uno predomina el grupo tetracarboxilporfirina (coproporfirina), mientras que en otros la mayor concentración de porfirinas corresponde al grupo octa carboxil-porfirinas (uroporfirinas) y heptacarboxil-porfirinas.

La Figura 4 muestra el espectro de IR de la copro, proto y uroporfirina (4A, 4B y $4 \mathrm{C}$ respectivamente) y de la mezcla de estas porfirinas con albúmina (Fig. 4D, E y $\mathrm{F}$ respectivamente). La Fig. 2C muestra un espectro de IR de una mezcla de copro, proto y uroporfirina.

La Figura 5 muestra la visión de un cálculo del grupo I por microscopía de polarización y la Figura 6 muestra el cálculo por microscopía electrónica de barrido.

La Tabla III muestra el contenido de iones de cuatro cálculos negros que contienen porfirinas frente a cuatro cálculos controles.

Se ha observado un aumento significativo del contenido de proteínas en los cálculos que contienen porfirinas frente a los cálculos controles $(1.75 \pm 0.95$ vs $0.39 \pm 0.4523 \mu \mathrm{g} / \mathrm{mg}$ de cálculo, $\mathrm{p}<0.0025)$. También, existe un aumento de la fracción lipídica en los cálculos que contienen porfirinas frente a los controles, así el colesterol es de 4,4 $\pm 3,93$ vs $0.002 \pm 0.041 \mu \mathrm{g} / \mathrm{mg}$ de cálculo, $\mathrm{p}<0.005$. Por otra parte, la suma de glicerol y triglicéridos es de $1.53 \pm 0.51$ vs $0.002 \pm 0.0027$ 
Tabla I

Contenido de carboxil-porfirinas en el grupo de cálculos negro (I), mancha negra (II), triamterene de color oscuro (III) y grupo control

\begin{tabular}{|c|c|c|c|c|c|c|}
\hline Grupo & 8-COOH & 7-COOH & 6-COOH & 5-COOH & 4-COOH & $2-\mathrm{COOH}$ \\
\hline Cálculo Negro como & 1,8 & - & - & - & 8,7 & - \\
\hline \multirow[t]{7}{*}{ Carbon (I) } & 12,2 & - & - & $\operatorname{Tr}$ & 57,99 & $\operatorname{Tr}$ \\
\hline & $\operatorname{Tr}$ & - & - & - & 2,71 & - \\
\hline & - & - & - & - & 25,0 & - \\
\hline & 5,4 & 2,95 & - & - & 5,15 & - \\
\hline & 25,5 & 11,0 & 4,99 & 3,85 & 13,0 & - \\
\hline & 332,9 & 70,7 & - & 9,93 & 20,6 & - \\
\hline & 35,2 & - & & - & - & - \\
\hline \multirow{4}{*}{$\begin{array}{l}\text { Mancha negra como el } \\
\text { Carbón (II) }\end{array}$} & 19,97 & 17,98 & 30,21 & - & 6,64 & - \\
\hline & 1,29 & 1,17 & 1,57 & 0,6 & 2,51 & - \\
\hline & - & - & - & - & - & - \\
\hline & 2,58 & 0,96 & 0,53 & - & 0,50 & - \\
\hline \multirow{7}{*}{$\begin{array}{l}\text { Triamterene de color } \\
\text { Oscuro (III) }\end{array}$} & - & - & - & - & 1,71 & - \\
\hline & - & - & - & - & $\operatorname{Tr}$ & - \\
\hline & - & - & - & - & 6,96 & - \\
\hline & - & - & - & - & 0,72 & - \\
\hline & - & - & - & - & 18,14 & - \\
\hline & 3,81 & - & - & - & 16,15 & - \\
\hline & 2,48 & 5,56 & $\operatorname{Tr}$ & - & 6,04 & - \\
\hline \multicolumn{7}{|l|}{ Control } \\
\hline STV & - & - & - & - & - & - \\
\hline WED & $\operatorname{Tr}$ & - & - & - & - & - \\
\hline STV & - & - & - & - & - & - \\
\hline WED & 0,38 & - & - & - & - & - \\
\hline CYST & - & - & - & - & - & - \\
\hline $\mathrm{STV}+\mathrm{A}$ & 0,14 & 0,04 & 0,02 & 0,02 & 0,05 & - \\
\hline $\mathrm{STV}+\mathrm{A}$ & - & - & - & - & - & - \\
\hline $\mathrm{STV}+\mathrm{A}$ & 0,06 & 0,03 & - & - & 0,09 & - \\
\hline A & 0,07 & - & - & - & $\operatorname{Tr}$ & - \\
\hline $\mathrm{UA}+\mathrm{WHE}$ & $\operatorname{Tr}$ & - & - & - & 0,17 & - \\
\hline WED & 0,4 & - & - & - & $\operatorname{Tr}$ & - \\
\hline WED + WHE & 0,3 & 0,14 & - & 0,03 & 0,2 & $\operatorname{Tr}$ \\
\hline WED & 0,53 & - & - & - & - & - \\
\hline UA & $\operatorname{Tr}$ & $\operatorname{Tr}$ & - & - & - & - \\
\hline UA & - & - & - & - & - & - \\
\hline UA & - & - & - & - & - & - \\
\hline UA & - & - & - & - & - & - \\
\hline WED & $\operatorname{Tr}$ & - & - & - & - & - \\
\hline STV & $\operatorname{Tr}$ & - & - & - & 0,09 & 0,60 \\
\hline STV & 0,6 & - & 0,13 & - & 0,06 & - \\
\hline UA & - & - & - & - & 0,31 & - \\
\hline
\end{tabular}

8-COOH: octacarboxil-porfirina (uroporfirina); 7- $\mathrm{COOH}$ : heptacaboxil-porfirina; 6- $\mathrm{COOH}$ : hexacarbosilporfirina; 5- $\mathrm{COOH}$ pentacarboxil-porfirina; 4- $\mathrm{COOH}$ : tetracarbosil-porfirina (coproporfirina); 2- $\mathrm{COOH}$ : dicarboxil-porfirina (protoporfirina). ST: Estruvita; WED: weddelita; WHE: whewellita; UA: ácido úrico; A: carbonato apatita; CYS: cistina.

$\mu \mathrm{g} / \mathrm{mg}$ cálculo, p<0.0125. En el espectro electroforético se ha observado la presencia de una banda que corresponde a la albúmina y se han detectado dos pequeñas bandas en la zona de la $\alpha$-globulina y el fibrinógeno ${ }^{8}$.

\section{DISCUSIÓN}

El aspecto morfológico de los cálculos de color negro con apariencia como el carbón (grupo I) es totalmente característico y difiere del resto de los cálculos renales ${ }^{3,6}$ En el trabajo publicado por el Dr. Cifuentes Delatte en $1984^{13}$ se indicó la existencia de una materia negra muy característica y no identificada, detectada en la cara cóncava de una serie de cálculos papilares. Es posible que estos cálculos puedan corresponder a los referidos como "cálculos de índigo"14. Thiel et al ${ }^{15}$ describieron cálculos renales como coágulos de sangre después del transplante renal. Los cálculos de triamterene (grupo III) mostraron un color oscuro ${ }^{4}$. Este hecho fue reportado por Ettinger et al (1917) que observaron una gama de colores en este tipo de cálculos que era desde el color típico dorado de estos cálculos a un color oscuro. Estos autores pensaron que este hecho era debido a la incorporación de pigmentos urinarios en los cálculos. $\mathrm{El}$ contenido de porfirinas en los cálculos negros y oscuros es sorprendentemente elevado, aunque encontramos un rango de valores muy variado.

En un intento de valorar el contenido de porfirinas en estos cálculos renales tan peculiares, se comparó con el contenido de porfirinas en hígado y eritrocitos, siendo éste de $1 \mathrm{ng} / \mathrm{mg}$ y $300 \mathrm{ng} / \mathrm{mg}$ respectivamente, correspondiendo a la coproporfirina ${ }^{6}$. En personas normales la eliminación en orina de coproporfirina representa alrededor del $70-80 \%$ de la eliminación total de porfirinas ${ }^{6}$.

Pacientes con hepatopatías pueden eliminar altas cantidades de porfirina, principalmente de coproporfirina (tetracarboxil-porfirina). En pacientes con algún tipo de porfiria o con fracaso renal 
Tabla II

Contenido de porfirinas en cálculos papilares oscuros

\begin{tabular}{|c|c|c|c|c|c|c|}
\hline Grupo & 8-COOH & 7-COOH & 6-COOH & 5-COOH & 4-COOH & $2-\mathrm{COOH}$ \\
\hline Cálculos & 0,99 & $\operatorname{Tr}$ & - & - & 2,12 & $\operatorname{Tr}$ \\
\hline Papilares & - & - & - & - & 3,9 & - \\
\hline \multirow[t]{19}{*}{ (IV) } & - & - & - & - & 1,3 & - \\
\hline & 0,21 & - & - & - & 0,12 & - \\
\hline & 3,89 & 0,60 & - & - & - & - \\
\hline & 0,99 & $\operatorname{Tr}$ & - & - & 2,12 & $\operatorname{Tr}$ \\
\hline & 0,43 & 0,36 & - & $\mathrm{Tr}$ & 0,40 & - \\
\hline & 1,93 & 1,59 & 0,71 & - & - & - \\
\hline & 0,67 & 0,44 & - & $\operatorname{Tr}$ & 0,36 & - \\
\hline & 0,76 & $\operatorname{Tr}$ & - & - & 0,71 & - \\
\hline & 0,66 & 0,49 & - & - & 0,26 & $\operatorname{Tr}$ \\
\hline & 1,51 & 1,88 & - & - & - & - \\
\hline & $\operatorname{Tr}$ & $\operatorname{Tr}$ & - & - & 0,50 & - \\
\hline & 11,53 & 11,72 & 3,01 & 1,05 & 1,54 & - \\
\hline & 6,45 & 6,94 & 6,45 & $\mathrm{Tr}$ & 8,01 & - \\
\hline & $\mathrm{Tr}$ & 41,28 & 76,84 & $\mathrm{Tr}$ & - & - \\
\hline & 73,41 & - & - & - & $\mathrm{Tr}$ & - \\
\hline & $\operatorname{Tr}$ & 5,6 & $\operatorname{Tr}$ & - & $\operatorname{Tr}$ & - \\
\hline & $\operatorname{Tr}$ & 8,35 & 6,05 & - & $\operatorname{Tr}$ & - \\
\hline & $\operatorname{Tr}$ & 5,85 & - & - & $\operatorname{Tr}$ & - \\
\hline & $\operatorname{Tr}$ & 4,71 & - & - & 1,42 & - \\
\hline
\end{tabular}

8-COOH: octacarboxil-porfirina (uroporfirina); 7-COOH: heptacaboxil-porfirina; 6-COOH: hexacarbosil-porfirina; 5-COOH: pentacarboxil-porfirina; 4-COOH: tetracarbosil-porfirina (coproporfirina); 2-COOH: dicarboxil-porfirina (protoporfirina)

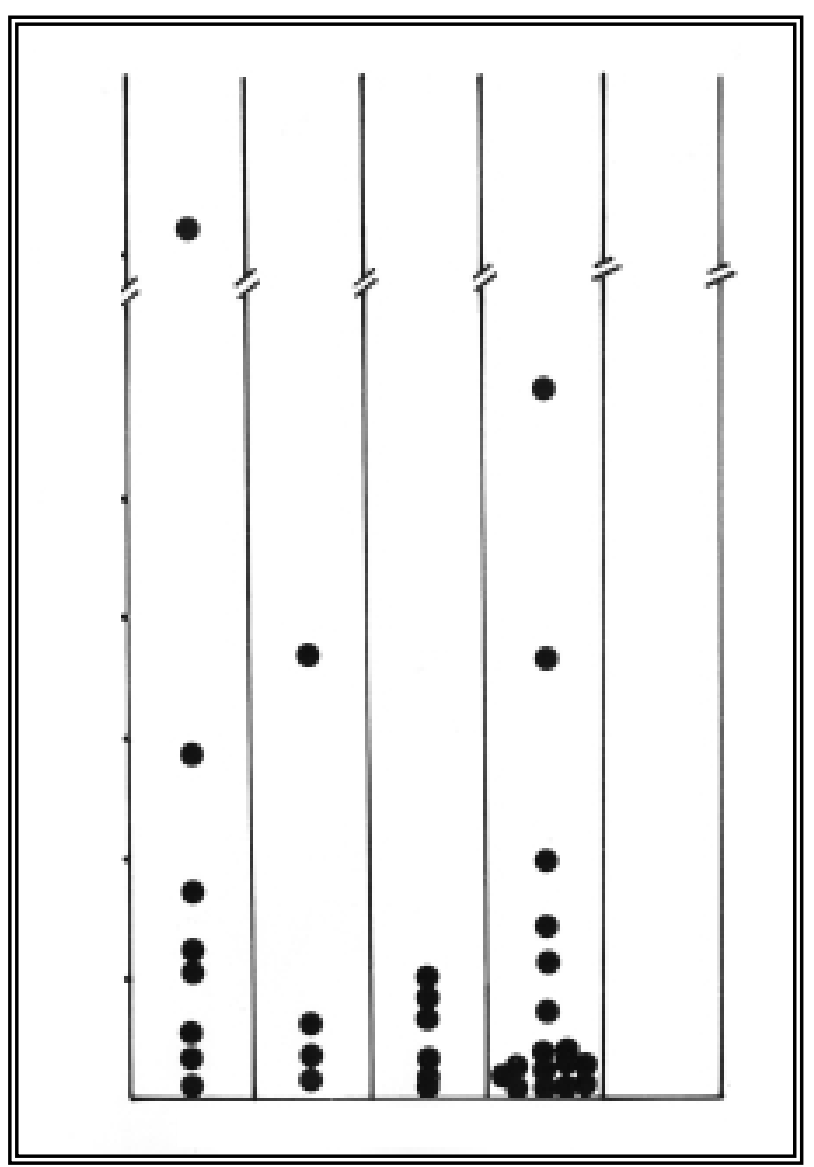

FIGURA 3. Contenido total de proteinas $(\mu \mathrm{g} / \mathrm{mg})$ en cálculos negros (I), cálculos con una mancha negra (II), cálculos negruzcos de triamterene (III), cálculos oscuros papilares (IV) y cálculos controles. crónico, la eliminación de porfirinas corresponde a las mas carboxiladas, así tenemos la uroporfirina (octacarboxil-porfirina) y heptacarboxil-porfirina. Precisamente en el tipo de cálculos negros y de color negruzco hemos encontrado estos dos patrones de acumulación de porfirinas. Durante el desarrollo del cálculo, el contenido de porfirinas puede haberse unido a otros componentes del cálculo. Es bien conocido que las porfirinas tienen afinidad para unirse a proteínas y lípidos ${ }^{8}$. Así, nosotros hemos encontrado un aumento significativo del contenido de proteínas y lípidos en los cálculos que contienen porfirinas ${ }^{8}$.

También es conocida la capacidad de unión de las porfirinas a iones para formar las metaloporfirinas. En los cálculos con porfirinas hemos encontrado un aumento significativo del contenido de $\mathrm{Fe}^{2+}$ y $\mathrm{Cu}^{2+}$ frente a los cálculos controles, no existiendo diferencias significativas entre el grupo de cálculos problema respecto al grupo control en el contenido de $\mathrm{Zn}^{2+}, \mathrm{Ca}^{2+}, \mathrm{Mg}^{2+}, \mathrm{K}+$, $\mathrm{Na}^{+}$y P. Los pacientes que expulsaron cálculos de triamterene padecían enfermedades hepáticas, hecho que puede explicar la alta concentración de coproporfirina ${ }^{4}$. Los pacientes que eliminaron cálculos negros mostraron que padecían un fracaso renal crónico, habiéndose encontrado en estos cálculos un predominio de uroporfirina. La hipótesis de que las porfirinas tuvieran un origen hematúrico no se ha podido confirmar, dado que la dicarboxil-protoporfirina (porfirina presente en los eritrocitos) no se ha detectado en estos cálculos.

Por otra parte, no tenemos referencias de la presencia de porfirinas en cálculos renales, excepto el encontrado por Castro-Mendoza ${ }^{18}$, en el que describe la existencia de coproporfirina III (Fig. 7) procedente de la parte oscura de un cálculo de xantina (Fig. 8). Miller et $\mathrm{al}^{18}$ reportaron el hecho de encontrar hematina en dos cálculos renales. 


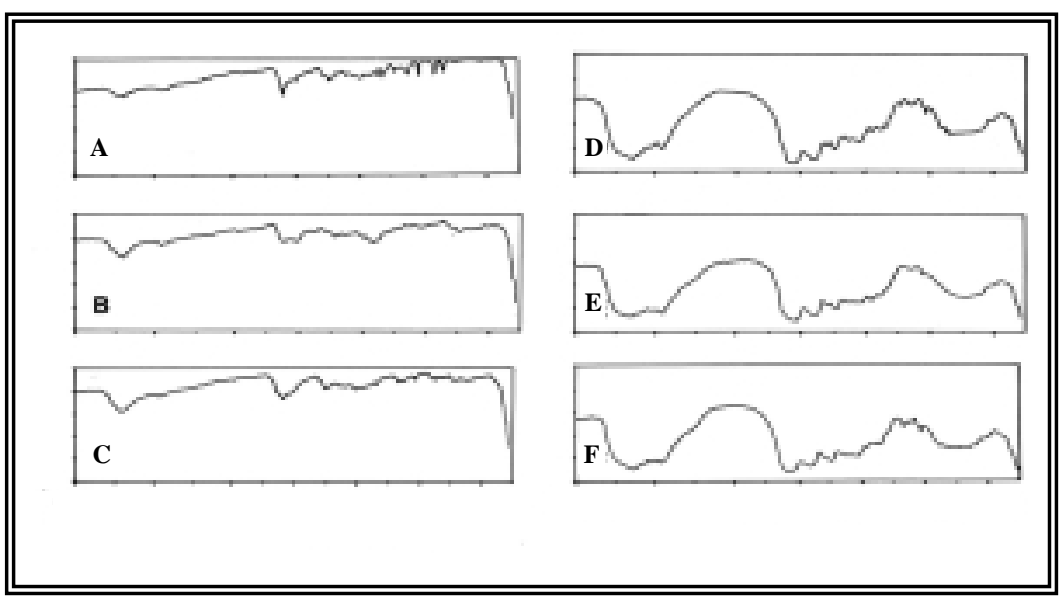

FIGURA 4. Espectros de infrarrojo de: estándar de copro, proto y uroporfirina (A, B, C, respectivamente). Espectros de infrarrojo correspondientes a la mezcla de copro, proto y uroporfirina con albúmina ( $D, E$ y $F$, respectivamente).
Escasa información puede obtenerse por espectroscopía infrarroja de la existencia y tipo de porfirina contenida en estos cálculos negros. Sin embargo, cuando el espectro de IR es el de "materia orgánica" en cálculos negros o negruzcos, es sugerente la existencia de porfirinas en ellos y debe completarse el estudio del cálculo con el análisis de porfirinas.

La alta concentración de porfirinas en este tipo peculiar de cálculos renales es difícil de evaluar. Son necesarios estudios posteriores para

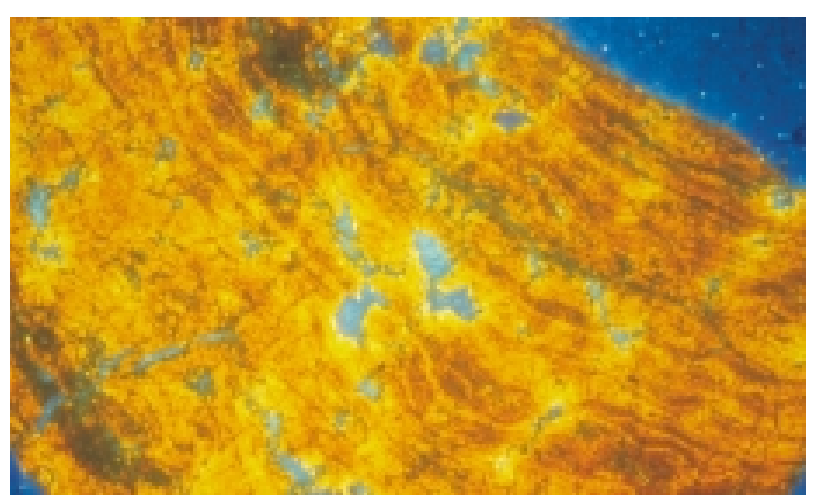

FIGURA 5. Imagen por microscopia de luz polarizada de un cálculo negro por el método de lámina delgada (aumento x 40).

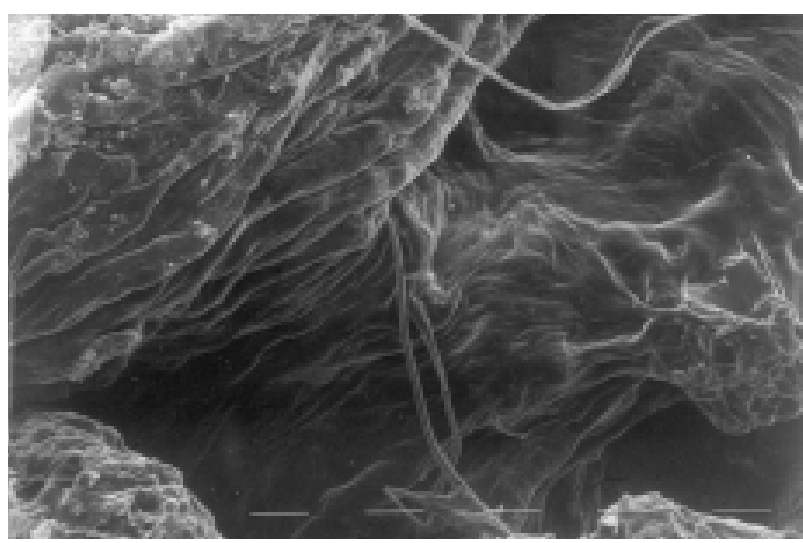

FIGURA 6. Imagen de un cálculo negro por microscopia electrónica de barrido (aumento $x \mathrm{80}$ ).

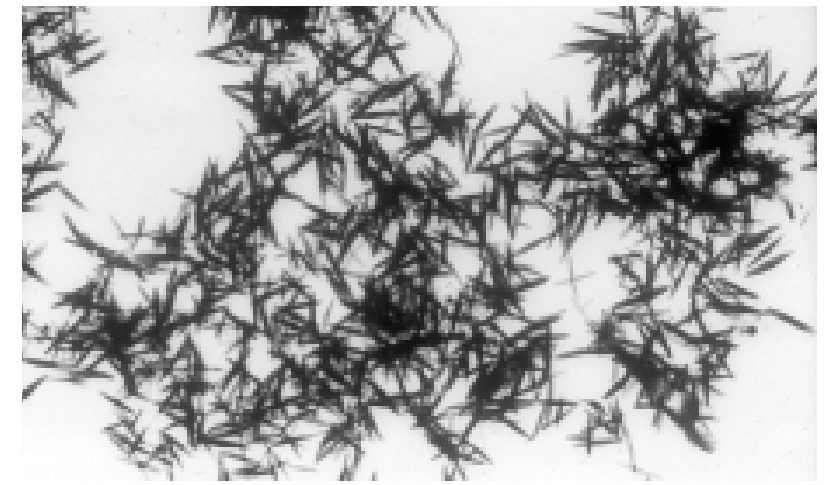

FIGURA 7. Coproporfirina III.

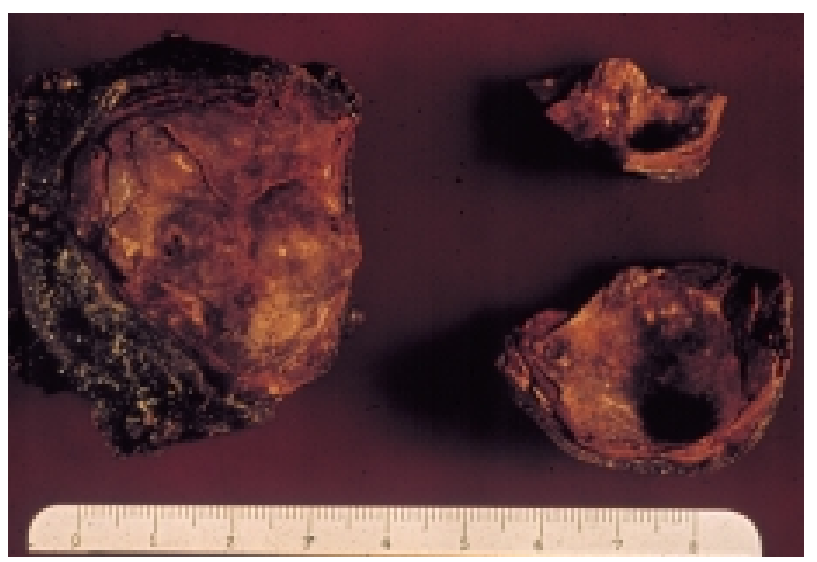

FIGURA 8. Cálculo de xantina donde puede observarse una mancha negra.

\section{Tabla III}

Contenido de componentes iónicos ( $\mathrm{\mu g} / \mathrm{mg}$ de cálculo) en cuatro cálculos negros y cuatro cálculos control

\begin{tabular}{lccccccccc}
\hline Cálculo & $\boldsymbol{N}^{\mathbf{o}}$ & $\boldsymbol{F e}^{2+}$ & $\mathbf{C u}^{2+}$ & $\mathbf{Z n}^{2+}$ & $\mathbf{C a}^{2+}$ & $\mathbf{M g}^{2+}$ & $\boldsymbol{K}^{+}$ & $\mathbf{N a}^{+}$ & $\boldsymbol{P}^{+}$ \\
Negros & 4 & $0.311 \pm 0.17$ & $0.043 \pm 0.026$ & $0.124 \pm 0.115$ & $81.75 \pm 114$ & $1.55 \pm 1.79$ & $20.05 \pm 29.5$ & Ind & $2.38 \pm 0.95$ \\
Controles & 4 & $0.053 \pm 0.02$ & $0.0008 \pm 0.003$ & $0.075 \pm 0.076$ & $185.2 \pm 44.6$ & $11.59 \pm 21.61$ & $0.89 \pm 0.77$ & $4.36 \pm 1.96$ & $39 \pm 61.5$ \\
& & $\mathrm{p}<0.0125$ & $\mathrm{p}<0.0125$ & NS & NS & NS & NS & NS \\
\hline
\end{tabular}


clarificar el mecanismo o mecanismos que conducen a las porfirinas a ser parte constituyente de cálculos renales.

\section{Agradecimientos}

Quiero hacer patente mi agradecimiento al Dr. Castro-Mendoza por haberme facilitado los dispositivos correspondientes a la coproporfirina III y el cálculo de xantina. Igualmente quiero agradecer al Dr. CifuentesDelatte el haber cedido el estudio de un cálculo negro por microscopía electrónica.

\section{REFERENCIAS}

1. Traba M, Rapado A. Componentes de la sangre en cálculos renales de "materia orgánica". Arch Esp Urol 1985;38:73-77.

2. Raba Ml, Perpiña J, Enriquez de Salamanca R. Análisis por espectrometría infrarroja de cálculos renales en cuya composición existen porfirinas. Actas Urol Esp 1987;3:309-312.

3. Traba Ml, Perpiña J, Enriquez de Salamanca R. Identificación y cuantificación de diversas porfirinas como componentes en un tipo peculiar de cálculo renal. Arch Esp Urol 1985;38:601-604.

4. Traba Ml, Perpiña J, Enriquez De Salamanca R. Porfirinas en cálculos de triamterene. Arch Esp Urol 1987;40:105106.

5. Days Rs, Enriquez de Salamanca R, Eales L. Quantitation of red cell porphyrin by fluorescence scanning after thin layer chromatography. Clin Chim Acta 1978;89:105-106.

6. Traba Ml, Perpiña J, Enriquez De Salamanca R. Porphyrin accumulation in some types of renal calculi. Med Sci Res 1988;16:1269-1271.

7. Traba Ml, Perpiña J, Enriquez de Salamanca R, Cifuentes Delatte L. Porphyrins as components of a certain kind of renal calculi. En: Pathogenese und klinik der harnsteine XII. Herausgegeben von W. Vahlensieck und G Gasser. Steinkopff-Verlag Darmtadt 1987;136-139.

8. Traba Ml, Arroyo F, Rubio M, Nuño J, Perpiña J, Enriquez De Salamanca R. Análisis de componentes iónicos, proteínas y lípidos en cálculos que contienen porfirinas. Actas Urol Esp 1987;(10):307-308.
9. Fiske Ch, Subbarow Y. Colorimetric determination of phosphorous. J Biol Chem 1925;66:375-379.

10. Bradford NM. A rapid and sensitive method for the quantitation of microgram quantities of protein. Anal Biochem 1976;72:248-254.

11. Richmond W. Preparation and properties of a cholesterol oxidase from Nocardia sp and its application to enzymatic assay of total cholesterol in serum. Clin Chem 1973:19 (12):1350-1356.

12. Bucolo G, David H. Quantitative determination of serum triglycerides by the use of enzymes. Clin Chem 1973;19 (5):476-82.

13. Cifuentes Delatte L, Medina JA, Miñón Cifuentes Jl. Cálculos papilares con placas atípicas. Arch Esp Urol 1984;37:569-576.

14. Heitzmann L. Urinary analysis and diagnosis by microscopical and chemical examination. Bailliere, Tindall and Cax, London, 1934; 128.

15. Thiel G, Brunn F, Enderlin F et al in: Urolithiasis Research. Fleisch H, Robertson WC, Smith LH and Vahlensieck W cols. Plenum Press, New York, 1981;131137.

16. Ettinger B, Oldroyd No, Slorgel F. Renal calculi in triamterene users. En: Urolithiasis clinical and basic research. Smith, Plenum Press, New York 1981;131-137.

17. Ettinger B, Oldroyd Na, Sorgel F. Triamterene nephrolithiasis. JAMA 1980;244(21):2443-2445.

18. Castro-Mendoza Hj. Xanthine-Coproporphyrin III. Adv Exp Med Biol 1980;122A:251-255.

19. Miller Ji, Mcbeath Rb, Drach Gw. Urinary hematín calculi. J Urol 1991;145(5):1043-1044.

Dra. M는. Traba Villameytide

Bioquímica Clínica

Sección Fisiopatología Ósea

Fundación Jiménez Díaz

Avda. Reyes Católicos, 2

28040 Madrid

(Trabajo recibido el 2 septiembre de 2004) 